\title{
Toward an accommodation account of deaccenting under nonidentity
}

\author{
Jeffrey Geiger \& Ming Xiang*
}

\begin{abstract}
Two competing models attempt to explain the deaccentuation of antecedentnonidentical discourse-inferable material (e.g., Bach wrote many pieces for viola. He must have LOVED string instruments). One uses a single grammatical constraint to license deaccenting for identical and nonidentical material. The second licenses deaccenting grammatically only for identical constituents, whereas deaccented nonidentical material requires accommodation of an alternative antecedent. In three experiments, we tested listeners' preferences for accentuation or deaccentuation on nonidentical inferable material in out-of-the-blue contexts, supportive discourse contexts, and in the presence of the presupposition trigger too. The results indicate that listeners by default prefer for inferable material to be accented, but that this preference can be mitigated or even reversed with the help of manipulations in the broader discourse context. By contrast, listeners reliably preferred for repeated material to be deaccented. We argue that these results are more compatible with the accommodation model of deaccenting licensing, which allows for differential licensing of deaccentuation on inferable versus repeated constituents and provides a principled account of the sensitivity of accentuation preferences on inferable material to broader contextual manipulations.
\end{abstract}

Keywords. deaccenting; prosody; information structure; givenness; inference; identity; accommodation; entailment

1. Introduction. It has long been recognized that there is a close connection between a constituent's information status within a discourse and its prosodic realization. Canonically, contentful discourse-new constituents are realized with a high or rising pitch accent (Chafe 1974, Pierrehumbert \& Hirschberg 1990). In English, this is most noticeable on the most embedded constituent, which receives a nuclear pitch accent, impressionistically the most prominent accent in the clause (Chomsky \& Halle 1968, Selkirk 1984, Büring 2016). For example, in (1), COFFEE exhibits a nuclear pitch accent, denoted by small caps.

(1) The caterer asked if there were any drinks to avoid, and I said I don't like COFFEE.

In contrast to new constituents, constituents with an identical correlate in a local linguistic antecedent tend not to exhibit a high pitch accent. Such constituents are exempt from the default rules of stress assignment via an operation called deaccenting or stress shift, and are realized as prosodically reduced, according to both listener judgments and a phonetic correlates of emphasis such as intensity, F0, and duration (Pierrehumbert \& Hirschberg 1990, Chodroff \& Cole 2019, Geiger \& Xiang 2019). Although the final coffee in (2) is in a structural position to receive a nuclear pitch accent, it is deaccented by virtue of the instance of coffee in the previous clause, and the nuclear accent falls instead on LIKE.

(2) The caterer asked if they should supply coffee, and I said I don't LIKE coffee.

*This work was supported by National Science Foundation Grant No. BCS-1827404. Authors: Jeffrey Geiger, University of Chicago (jeffrey.geiger@ pomona.edu) \& Ming Xiang, University of Chicago (mxiang@uchicago.edu). 
This mapping between givenness and deaccentuation exhibits a number of complications, however. One is that constituents are typically deaccented only when they are in a structural position isomorphic to that of their antecedent (cf. \#Mary saw John, then KIM saw Mary; Tancredi 1992, Schwarzschild 1999). This is not the primary focus of the current paper, but is an important consideration in the grammar of deaccentuation.

A second complication is that the literature recognizes a number of cases in which a constituent can felicitously be deaccented despite not having an identical correlate in a local linguistic antecedent. This includes constituents that corefer with an antecedent, as in (3); constituents that are entailed by an antecedent constituent, modulo existential closure, as in (4); constituents whose meanings are made salient by synthesizing information from the antecedent with broader world knowledge, as in (5); and constituents whose meanings are made salient by the nonlinguistic context, as in (6).

(3) A: Did you see Dr. Cremer to get your root canal?

B: Don't remind me. I'd like to STRANGLE the butcher.

(Büring 2007)

(4) Bach wrote many pieces for viola. He must have LOVED string instruments. (van Deemter 1999)

(5) First John called Mary a Republican, and then SHE insulted HIM. (Lakoff 1968)

(6) [Hearer cocks their head to one side as if listening for a faint or distant noise.] Speaker: I heard it, TOO.

(Rochemont 1986)

The diverse configurations that give rise to deaccentuation, including under identity, as in (2), and under nonidentity, as in (3) through (6), raise the question of how to characterize the set of deaccentable material, and what combination of grammatical and extragrammatical mechanisms generates deaccented structures. Two classes of solution have emerged in the literature.

The first approach, which for convenience we refer to as the grammatical model of deaccentuation licensing, concentrates on developing a single condition under which deaccenting is licensed; this condition accounts for the deaccentuation of both antecedent-identical and -nonidentical material. The fundamental insight of the grammatical approach is that givenness is the underlying property that characterizes deaccentable material, and that constituents with identical antecedents merely constitute a trivial subset of given material.

Some grammatical approaches posit essentially a one-to-one mapping between givenness and deaccentuation, with the challenge lying in determining what material counts as given. For instance, Rochemont (1986) exempts constituents from focus marking when they are $c$-construable, a flexible notion of givenness that allows deaccentuation of material entailed by an antecedent, salient in the nonlinguistic context, or treated as background information by the speaker and hearer. Van Deemter $(1994,1999)$ suggests that a constituent can be deaccented when it is object-given, meaning that it corefers with an antecedent, or concept-given, when it is entailed by an antecedent. Baumann and Riester (2012) make a similar appeal in their RefLex annotation scheme, where a 
constituent can be "referentially" ( $\mathrm{r}$-)given if it corefers with an antecedent, or "lexically" (1-)given if it is identical to or entailed by an antecedent via synonymy or a superset relation.

Other grammatical approaches interface more directly with focus theory, and aim to simultaneously capture the structural constraints on deaccentuation and the mapping between notional givenness and deaccenting. One such account comes from Rooth (1992). Under this model, the use of any focus-marked structure introduces a presupposition that there is a focus alternative to the structure that is contextually available. This requirement is evaluated along semantic lines, meaning that non-F-marked (deaccented) material is felicitous when it is entailed by an antecedent, whether or not the constituents are string-identical (e.g., string instruments with an antecedent of viola in (4)). In another influential model, Schwarzschild (1999) requires that every substructure of a sentence be GIVEN, meaning informally that the context supplies a meaning that entails the constituent with its F-marked components replaced by variables. As in Rooth's account, the requirement that deaccented material be merely entailed by the antecedent allows for the deaccenting of material that does not necessarily have a string-identical correlate in the antecedent. Crucially, in both accounts, antecedent-identical constituents are deaccentable because they are trivially entailed by their identical antecedent.

In contrast to the grammatical approach, the accommodation model evokes two separate mechanisms for generating deaccentuation on antecedent-identical constituents versus nonidentical constituents. According to the accommodation model, the grammatical property characterizing deaccentable material is string identity with an antecedent, not givenness. Thus, deaccentuation is licensed on antecedent-identical constituents using this grammatical mechanism. By contrast, deaccentuation of antecedent-nonidentical material, as in (3) through (6), is strictly ungrammatical. However, such instances of deaccentuation can be marked acceptable via a second, extragrammatical mechanism. If it is reasonable to do so, an alternative antecedent can be accommodated that contains an identical correlate to the deaccented material; the deaccenting is then treated as acceptable on the basis of identity with this accommodated antecedent.

In an early accommodation approach, Tancredi (1992) proposes that deaccentuation requires prior "instantiation" in the antecedent (string identity), but that the linguistic context can be augmented via "pragmatic incrementation" to include implied alternative utterances that would license observed instances of deaccentuation under nonidentity. Fox (2000) similarly characterizes non-Fmarked (deaccented) material without an identical correlate in the antecedent as accommodationseeking material, meaning its use triggers the accommodation of an alternative linguistic structure that would have licensed deaccentuation according to the grammatical identity requirement. Finally, while Wagner (2012) does not concentrate on an accommodation operation, he makes reference to how such a mechanism could underlie cases of deaccentuation under nonidentity.

Despite sustained interest in the connection between givenness and deaccentuation, there has been little systematic quantitative research on the prosodic properties of inferable (discourseaccessible) material, such as constituents entailed by, but not identical to, an antecedent. Two recent exceptions include Chodroff \& Cole (2019) and Geiger \& Xiang (2019), who examined the production of new, given, and inferable nouns and verbs, respectively. Each found that discourseaccessible constituents tend to be realized more similarly to discourse-new than given material, casting doubt on the assumption in both the grammatical and accommodation literature that deac- 
centing under nonidentity is straightforward.

The present paper builds on these production studies by examining listener assessments of prosodic naturalness for new, repeated, and inferable verbs in perception. Whereas it is possible that deaccentuation is optional in production, or that speakers in the prior production studies may not have been aware of the relevant inferencing relations making the critical constituents inferable, listeners in a perception study must contend with the fact that the speaker chose to accent or deaccent a particular constituent. This may lead to more robust conclusions about whether deaccentuation is licensed on inferable constituents, and about what licensing mechanisms underlie deaccentuation for antecedent-identical and -nonidentical material.

2. Experiment 1. Experiment 1 assessed the naturalness of accentuation and deaccentuation on verbs of three different levels of information status: new, inferable, and repeated (given). The goal was to compare listeners' prosodic preferences for inferable verbs to those for new and repeated verbs, which canonically should sound more natural when they are accented and deaccented, respectively. Crucially, the grammatical licensing model predicts that highly inferable and repeated verbs should exhibit roughly the same behavior, since they are subject to the same constraint on deaccenting, while the accommodation model leaves room for differential behavior between inferable and repeated verbs, since they are treated by separate deaccenting licensing mechanisms.

2.1. DeSign AND MATERIALS. The critical materials in the experiment were audio-recorded sentences of the form $S V O$ and $S V O$. The full experiment had a 3 (verb status) $\times 2$ (object status) $\times 2$ (accent) design. Within each item, the second SVO string remained constant, while the first SVO clause varied to determine the discourse status of the second-clause constituents. All subjects and objects were proper names, and the second-clause subject was always different from the firstclause subject. Table 1 outlines the conditions discussed in this paper, which include the full verb status and accent manipulations, but only one level for object status.

\begin{tabular}{lll} 
Accent & Verb status & Recording \\
\hline \multirow{3}{*}{ Accented } & New & Gabriel punished Amy, and Nan SURPRISED Amy. \\
& Inferable & Ethan astounded Amy, and Nan SURPRISED Amy. \\
& Repeated & Benjamin surprised Amy, and Nan SURPRISED Amy. \\
\hline \multirow{3}{*}{ Deaccented } & New & Gabriel punished Amy, and NAN surprised Amy. \\
& Inferable & Ethan astounded Amy, and NAN surprised Amy. \\
& Repeated & Benjamin surprised Amy, and NAN surprised Amy.
\end{tabular}

Table 1: Sample Experiment 1 stimuli. Small caps: nuclear accent. Italics: deaccentuation.

The second-clause verb could be new, repeated, or inferable. New verbs did not stand in a clear inferencing relationship with the first-clause verb, such as surprised following punished. Repeated verbs were identical to the first-clause verb, as in surprised following surprised. Inferable verbs stood in a nonidentical inferencing relation to the antecedent verb, such that they were discourse-accessible, but not given. Two such inferencing relations were tested, each in one half of the experimental items: entailment (e.g., astounded-surprised), and more informal semantic relatedness that might support the conclusion that the second verb was accessible despite not being entailed by the first (e.g., charmed-seduced). The meanings of the inferable verbs were rated as highly available in the context of their antecedents in a separate norming study, whereas inferabil- 
ity ratings for the new verbs were low (Geiger \& Xiang 2019). ${ }^{1}$ For the experiments presented here, the results for the two types of inferencing relations were qualitatively very similar, so we collapsed the two categories and the results are presented together as one inferable condition.

The second-clause object could be old or new. Old objects were identical to the object of the first clause, while new objects were a different proper name not used anywhere else in the sentence. Since an old object should canonically be deaccented and a new object accented, this manipulation determined whether the second-clause verb was in nuclear (preceding an old object) or prenuclear (preceding a new object) position. Ratings did not reliably vary as a function of either verb status or accent in the new-object conditions, so only the results from the old-object conditions are presented and discussed in this paper.

Finally, the second-clause verb could be accented or deaccented. Two naive participants, one female and one male, read aloud SVO and SVO sentences featuring the verb status $\times$ object status manipulation described above (for more information, see Geiger \& Xiang 2019). In this paradigm, new verbs canonically should be accented, while repeated verbs should be deaccented. Thus, to generate sentences with the appropriate second-clause verb accent, recordings of second clauses (including and) with typical accentuation (read as new) or typical deaccentuation (read as repeated) were cross-spliced with the appropriate first-clause recordings to generate sentences with each verb status/object status combination.

2.2. Procedure. Participants were gathered via Amazon Mechanical Turk with the stipulations that they be at least 18 years old, native speakers of English, and using a computer in the United States. Upon accepting the task, participants were redirected to the experiment, conducted in Ibex Farm (Drummond 2020). They provided informed consent, verified that the experiment site played sound at an appropriate volume on their system, filled out a demographic survey, and completed two practice trials to familiarize them with the setup and response protocol.

In each critical trial, participants first viewed a preview screen that displayed, as text, the sentence they would be rating. Participants then pressed any key to advance from the preview screen to the test screen. Upon advancing, the audio file played automatically over the participant's speakers. Participants were prompted on this screen to rate how natural they found the "melody" or "tune" of the sentence, where 1 represented the least natural and 7 represented the most natural.

The experiment consisted of 24 critical trials as well as 10 filler trials featuring 5 canonical and 5 noncanonical productions of English prosodic contours (e.g., declaratives, polar questions, lists). The experiment took approximately 8 minutes to complete.

2.3. PARTICIPANTS. 142 participants (67 female, mean age 36.6 years) took part in the experiment. The data from one participant was excluded because they failed to identify themselves as a native speaker of English, and the data from a further five participants was excluded due to inattention (mean response time less than 1000 milliseconds).

2.4. Results AND ANAlysis. The results of Experiment 1 are shown in Figure 1. A visual inspection of the plot shows that as expected, participants rated sentences with new second-clause

\footnotetext{
${ }^{1}$ Sample prompt: Suppose you know that Alice astounded Billy. How likely do you think it is that Alice surprised Billy? The suppose sentence contained the first-clause antecedent verbs, conditioning either a new or inferable relation, while the rating question contained the target second-clause verb. Mean inferability score for inferable verbs: $6.14 / 7$. Mean score for new verbs: 2.14/7.
}

Jeffrey Geiger and Ming Xiang:

Toward an accommodation account of deaccenting under nonidentity. 
verbs as sounding more natural if that verb was accented than if it was deaccented. Conversely, sentences with repeated verbs received higher ratings when the verb was deaccented. Crucially, the results for inferable verbs resemble those for discourse-new verbs, with participants preferring accentuation over deaccentuation.

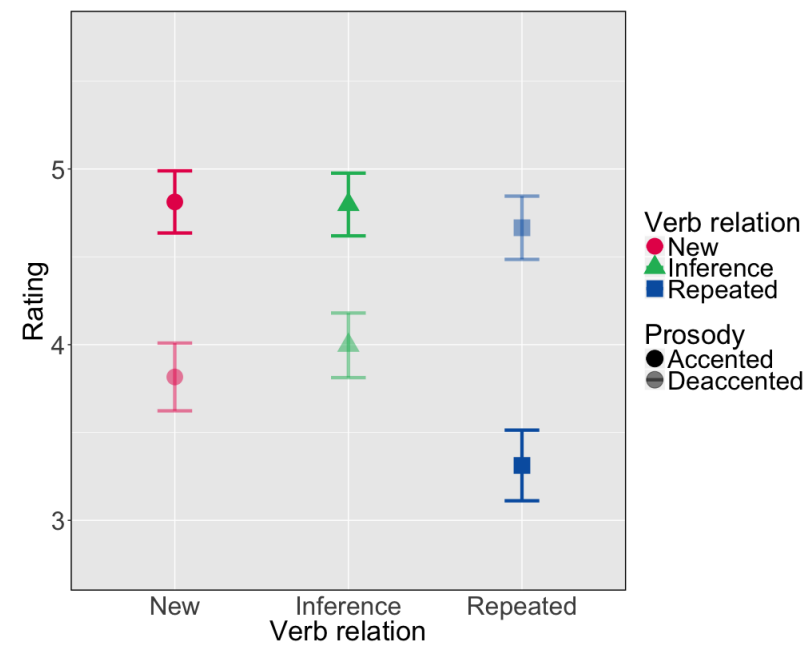

Figure 1: Experiment 1 results. Error bars: 95\% confidence interval.

To explore the results in further detail, they were fit to a linear mixed-effects regression model with an interaction of verb status and accent, main effects of verb status and accent, and random intercepts for participant and item. ${ }^{2}$

There was a significant interaction between verb status and accent $(\mathrm{p}<.001)$, and the main effects of verb status $(\mathrm{p}<.001)$ and accent $(\mathrm{p}<.05)$ were significant. Paired comparisons were carried out using estimated marginal means to test for an accentuation preference within each verb status level (i.e., new, inference, repeated). These paired comparisons indicated that the ratings of accented- versus deaccented-verb sentences differed significantly within all three levels of verb status (p's $<.001)$.

2.5. DISCUSSION. The paired comparisons indicated that participants significantly preferred sentences where new verbs were accented rather than deaccented, and they preferred sentences where repeated verbs were deaccented rather than accented. These results are unsurprising, as they conform to the canonical accent patterns for new and given material in nuclear position.

Crucially, participants significantly preferred sentences in which inferable verbs were accented over those in which they were deaccented. This contrasts somewhat with the predictions of both the grammatical and the accommodation accounts of deaccenting under nonidentity. Both models are meant to generate deaccentuation of inferable material, including entailed constituents. By contrast, the experimental results indicate that participants tend to treat inferable constituents as though they are discourse-new, with accentuation reliably preferred over deaccentuation.

Despite the mismatch between the experimental results and the prediction made by both accounts that deaccentuation of inferable material should be acceptable, the results are less problematic for the accommodation account than for the grammatical account. The grammatical model

\footnotetext{
${ }^{2}$ Imer model specification: Response $\sim$ VerbStatus * Accent $+(1 \mid$ Participant $)+(1 \mid$ Item $)$.
} 
uses one mechanism to generate deaccenting, with deaccenting under identity merely a subset of the cases in which the givenness requirement holds. Thus, it is not clear that this model can predict such a wide gap in (de)accentuation preferences for inferable versus repeated material.

By contrast, the accommodation model uses two mechanisms to generate deaccenting for inferable versus repeated material. The preference for accentuation on inferable constituents is somewhat at odds with the intended prediction of the model. However, since deaccentuation of inferable material is generated by an extragrammatical accommodation mechanism, whereas deaccentuation of repeated material is triggered by identity with an antecedent, the differential accentuation preferences for inferable and repeated material are not as clearly problematic for this model.

3. Experiment 2. Experiments 2 and 3 focus on further exploring the feasibility of the accommodation account. This model proposes that deaccenting under nonidentity relies on the extragrammatical accommodation of an alternative antecedent that would license deaccenting under identity. The pragmatic nature of this mechanism suggests that it should be sensitive to manipulations in the broader discourse context that might encourage or discourage such accommodation.

Whereas Experiment 1 presented the critical sentences out of the blue, Experiment 2 explored the role of the broader discourse by introducing a supportive context beyond the critical sentence. If the broader context supports parallel readings for inferable verbs and their antecedents, participants might find it more natural to accommodate the necessary alternative antecedent according to the accommodation model, increasing ratings for tokens with deaccented inferable verbs.

3.1. Design, MATERIAls, AND PROCEDURE. The design, materials, and procedure were identical to those for Experiment 1, with one modification. In Experiment 2, the preview screen on each trial now displayed a context sentence instead of a text representation of the sentence the participant would hear. Participants were told that the sentence on the warning screen represented a scenario, and that the sentence they heard represented someone talking about the scenario.

The purpose of the context sentence was to promote a "pragmatically identical" reading of the inferable verb and its antecedent. For instance, for the critical inferable-verb sentence Veronica bullied Roy, and Kendall intimidated Roy, the context sentence was As they did every year, the teachers worried about how the students would interact with each other on the first day of high school. The purpose of the context sentence here is to suggest that bullying and intimidation represent situationally comparable actions in the context - both are clearly negative interactions, and they do not obviously contrast with one another.

3.2. PARTICIPANTS. 144 participants (53 female, mean age 33.5) took part in Experiment 2. The data from two participants was excluded from analysis because they failed to self-identify as native English speakers, while the data from a further seven participants was excluded due to inattention.

3.3. Results And AnAlysis. The results of Experiment 2 are shown in Figure 2. As in Experiment 1 , the plot appears to suggest a reliable preference for accentuation on new verbs and deaccentuation on repeated verbs. Accentuation is once again preferred for inferable verbs, but the magnitude of this preference appears to be reduced relative to Experiment 1.

The initial analysis conducted for Experiment 2 was identical to that for Experiment 1. The linear mixed-effects regression model showed a significant interaction of verb status and accent $(\mathrm{p}<.001)$. The main effect of verb status was significant $(\mathrm{p}<.001)$, while the main effect of accent 


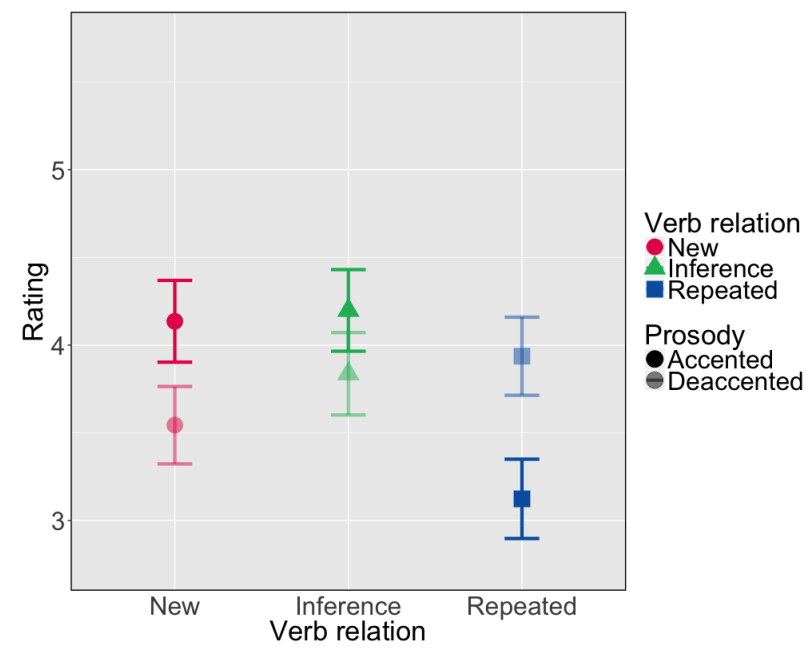

Figure 2: Experiment 2 results. Error bars: 95\% confidence interval.

was not $(\mathrm{p}>.5)$. Paired comparisons using estimated marginal means indicated that accented and deaccented tokens received significantly different ratings within all three levels of verb status (new $\mathrm{p}<.001$, inferable $\mathrm{p}<.01$, repeated $\mathrm{p}<.001$ ).

To compare the magnitude of the preference for accentuation on inferable verbs in each experiment, the inferable-verb data from Experiments 1 and 2 was fit to a linear mixed-effects regression model with an interaction of experiment and accent, main effects of experiment and accent, and random intercepts for item and participant. ${ }^{3}$ The interaction of experiment and accent was significant $(\mathrm{p}<.05)$, as were the main effects of experiment $(\mathrm{p}<.01)$ and accent $(\mathrm{p}<.001)$.

3.4. Discussion. As in Experiment 1, the paired comparisons for the Experiment 2 results indicated an expected preference for accentuation on new verbs and for deaccentuation on repeated verbs. For inferable verbs, there was still a preference for accentuation over deaccentuation. However, the experiment-accent interaction in the second model indicates that the preference for accentuation on inferable verbs was significantly reduced in magnitude in Experiment 2 compared to Experiment 1. This finding is compatible with the accommodation account, with broad contextual support for an identical reading of inferable verbs and their antecedents contributing to participants' willingness to accommodate the appropriate antecedent to license deaccenting. By contrast, it would be difficult for the grammatical account to explain such a difference between the two experiments, since the norming study indicated that participants found inferable verbs to be highly available even in the out-of-the-blue contexts of Experiment 1, and this type of givenness is the sole determiner of accent according to the grammatical model.

4. Experiment 3. Like Experiment 2, Experiment 3 explores how manipulations in the discourse context outside the critical constituents can affect listeners' judgments of the naturalness of accentuation and deaccentuation on inferable constituents. Where Experiment 2 explored the effect of adding information supporting deaccentuation to the broad discourse context, Experiment 3 tests the effect of adding the presupposition trigger too to the end of each stimulus sentence.

\footnotetext{
${ }^{3}$ Imer model specification: Response $\sim$ Experiment $*$ Accent $+(1 \mid$ Item $)+(1 \mid$ Participant $)$.
} 
On an informal level, too should indicate that the content of the second clause builds in some way on the discourse contribution of the first clause (Beaver \& Clark 2008). Similarly to the role played by the supportive context in Experiment 2, this might promote readings of the critical sentence where inferable verbs and their antecedents are treated as "situationally identical", indicating instances of identical or comparable events rather than contrasting or unrelated ones. In turn, this might encourage accommodation of the necessary alternative antecedent to license deaccenting, increasing the ratings for sentences with deaccented inferable verbs.

4.1. Design, MATERIAls, AND PROCEDURE. The design, materials, and procedure were identical to Experiment 1, except that each critical sentence was augmented with the word too at the end of the second clause (e.g., Ethan astounded Justin, and Nan surprised Amy, too). This addition necessitated re-recording the stimuli, as too was not present in the original recordings. The same female speaker who recorded half of the Experiment 1 stimuli re-recorded all of the necessary sentences using the production paradigm described in Geiger \& Xiang (2019) with too added to the end of each critical sentence. The clauses of these recordings were then cross-spliced as in Experiment 1 to create the same $3 \times 2 \times 2$ design (only old-object trials reported here).

4.2. PARTICIPANTS. 140 participants (46 female, mean age 37.0 years) took part in Experiment 3. The data from 23 participants was excluded from analysis due to inattention.

4.3. Results And AnAlysis. The results of Experiment 3 are shown in Figure 3. Once again, the plot suggests a preference for deaccentuation on repeated verbs. In contrast to the prior results, however, there appears to be no preference between accentuation and deaccentuation on new verbs. Finally, for inferable verbs, deaccentuation appears to be preferred for the first time.

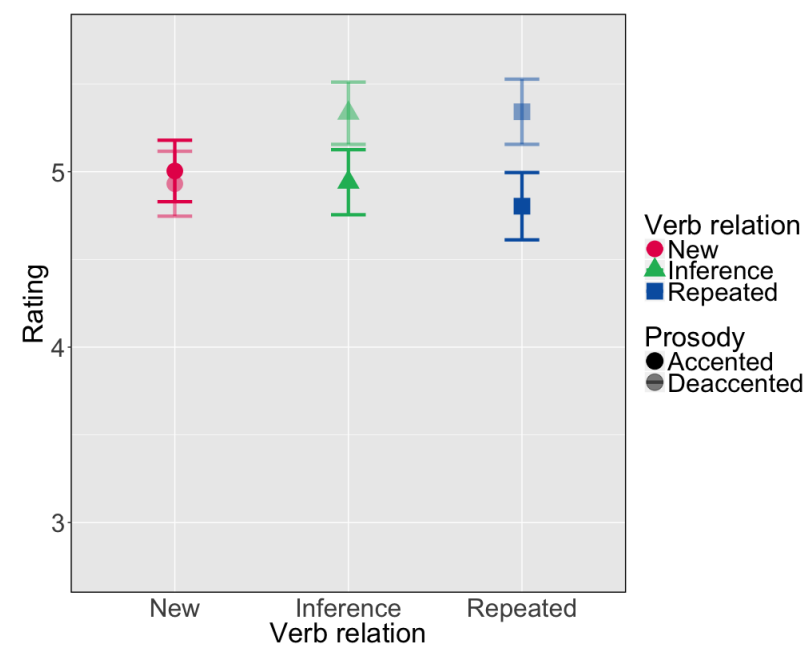

Figure 3: Experiment 3 results. Error bars: 95\% confidence interval.

The analysis for Experiment 3 was identical to those for the previous experiments. The linear mixed-effects regression model showed a significant interaction of verb status and accent $(\mathrm{p}<.001)$. The main effect of accent was significant $(\mathrm{p}<.001)$, while the main effect of verb status was not significant $(\mathrm{p}<.1)$. Paired comparisons indicated that accented- and deaccented-verb trials received significantly different ratings within the inferable and repeated levels for verb status (p's $<.001)$, 
but not within the new level ( $\mathrm{p}>.4)$.

4.4. DisCUSSION. The addition of too had a substantial impact on ratings compared to the results for Experiment 1. Most critically, the previous reliable preference for accentuation on inferable constituents gave way to a reliable preference for deaccentuation. This suggests that too promoted a reading where the inferable-verb second clause builds on the contribution of the first clause, encouraging treatment of the inferable verbs as "given" and, by extension, deaccentuation.

Interestingly, while the canonical preference for deaccentuation of repeated verbs persisted, there was no longer a reliable preference for accenting new verbs. This may be connected to the impressionistic observation that the rating "floor" in this experiment was higher than in the previous experiments; even relatively "bad" prosodic configurations received fairly high ratings. This suggests that the addition of too had substantial ameliorative effects on naturalness judgments across the board, indicating that the results of this experiment should be interpreted with caution.

Nevertheless, the results of Experiment 3 appear to support the accommodation model, with the addition of too suggesting that inferable verbs should be treated as though they were given, namely, be deaccented. This indicates that, contra the treatment of the accommodation account in the literature, it may not be sufficient for hearers merely to encounter a deaccented inferable verb for it to be marked as acceptable, but that such instances can be found acceptable in the presence of additional mitigating factors, such as presupposition triggers. By contrast, it is once again unclear how the grammatical model would account for the difference in behavior in the inferable conditions of Experiments 1 and 3, since the addition of too does not clearly affect the predictions of this model when the verbs in question were already highly inferable in Experiment 1.

5. General discussion. Considered together, the results of the three experiments are more compatible with the dual-mechanism accommodation account of deaccenting under nonidentity than the single-mechanism grammatical account. Both models are intended to generate canonically cited examples of deaccented antecedent-nonidentical material, such as constituents coreferring with or entailed by an antecedent. By contrast, Experiment 1 indicated that listeners preferred for highly inferable material, including both entailed verbs and verbs linked to their antecedents by more informal inferencing relations, to be accented as though they were discourse-new.

While this finding is at odds with the goal that both models should generate deaccenting under nonidentity, it is more problematic for the grammatical account than the accommodation account. The reason for this is the substantial difference between the preference for accentuation on inferable constituents and the preference for deaccentuation on repeated constituents. The grammatical model uses a single mechanism - givenness marking, or a structural constraint that subsumes both inferable and repeated material - to determine which constituents must be deaccented. Because of this unified constraint, it is not clear how the grammatical model would make such radically different predictions for the inferable conditions versus the repeated conditions.

Unlike the grammatical model, the accommodation model uses two distinct mechanisms to generate deaccenting on inferable and repeated material. Deaccentuation of repeated material is derived grammatically by virtue of string identity with an antecedent. Deaccentuation of inferable material is generated via an extragrammatical process of accommodation of an alternative antecedent containing an identical correlate to the deaccented material. The fact that there are separate mechanisms for inferable and repeated material means that the differential pattern of results 
in these two conditions is not fundamentally incompatible with the accommodation model.

Experiments 2 and 3 tested the degree to which listeners' preferences for accentuation or deaccentuation on inferable verbs were sensitive to manipulations in the broader discourse context. In Experiment 2, the addition of a broader context supporting a "situationally identical" reading for inferable verbs and their antecedents decreased the magnitude of the preference for accentuation on inferable verbs. In Experiment 3, addition of the presupposition trigger too reversed this preference to a preference for deaccentuation on inferable verbs.

Since the inferable verbs were already rated in the norming study as highly available even in the out-of-the-blue contexts, it is not clear how the grammatical model would explain the effect of these contextual manipulations on accent preferences. By contrast, the accommodation model allows for more holistic assessment of the discourse at large, with listeners potentially considering the entire context to determine whether the required accommodation is reasonable. Deaccenting of inferable verbs was not rated as acceptable in out-of-the-blue contexts, but this pattern of results is not categorically ruled out by the accommodation model. Further, the pragmatic mechanisms by which a supportive discourse context or a presupposition trigger could promote the necessary accommodation operation are relatively clear.

6. Conclusion. In three experiments, we investigated listeners' preferences for accentuation or deaccentuation on discourse-accessible or "inferable" verbs, and compared these preferences to the better-understood preferences for discourse-new and repeated constituents. Contra commonly cited examples, deaccentuation of inferable constituents was rated as less natural than accentuation in out-of-the-blue contexts. This preference was mitigated somewhat with the addition of a discourse context supporting parallel readings of inferable verbs and their antecedents, and reversed to a preference for deaccentuation with the addition of the presupposition trigger too. We argued that the dual-mechanism accommodation model of deaccenting licensing is more compatible with the results of all three experiments than the single-mechanism grammatical account. Future research exploring other aspects of this problem, such as the time course according to which accommodated inferences develop, may further confirm this conclusion.

\section{References}

Baumann, Stefan \& Arndt Riester. 2012. Referential and lexical givenness: Semantic, prosodic and cognitive aspects. In Gorka Elordieta \& Pilar Prieto (eds.), Prosody and Meaning, 1-34. Berlin: Mouton de Gruyter. https://doi.org/10.1515/9783110261790.119.

Beaver, David I. \& Brady Z. Clark. 2008. Sense and Sensitivity: How Focus Determines Meaning. Malden, MA: Wiley-Blackwell. https://doi.org/10.1002/9781444304176.

Büring, Daniel. 2007. Semantics, intonation and information structure. In Gillian Ramchand \& Charles Reiss (eds.), The Oxford Handbook of Linguistic Interfaces, 445-474. Oxford: Oxford University Press. https://doi.org/10.1093/oxfordhb/9780199247455.013.0015.

Büring, Daniel. 2016. Intonation and Meaning. Oxford: Oxford University Press. https://doi.org/10.1093/acprof:oso/9780199226269.001.0001.

Chafe, Wallace L. 1974. Language and consciousness. Language 50(1). 111-133. https://doi.org/10.2307/412014.

Chodroff, Eleanor \& Jennifer Cole. 2019. The phonological and phonetic encoding of information 
structure in American English nuclear accents. In Sasha Calhoun, Paola Escudero, Marija Tabain \& Paul Warren (eds.), Proceedings of 19th International Congress of Phonetic Sciences, Melbourne, Australia 2019, 1570-1574. Canberra, Australia: Australasian Speech Science and Technology Association Inc.

Chomsky, Noam \& Morris Halle. 1968. The Sound Pattern of English. New York: Harper and Row.

van Deemter, Kees. 1994. What's new? A semantic perspective on sentence accent. Journal of Semantics 11. 1-32. https://doi.org/10.1093/jos/11.1-2.1.

van Deemter, Kees. 1999. Contrastive stress, contrariety, and focus. In Peter Bosch \& Rob van der Sandt (eds.), Focus: Linguistic, Cognitive, and Computational Perspectives, 3-17. Cambridge: Cambridge University Press.

Drummond, Alex. 2020. Ibex 0.3.7 Manual. https: / / github. com/addrummond/ibex/ $\mathrm{blob} / \mathrm{master/docs/manual.md.}$

Fox, Danny. 2000. Economy and Semantic Interpretation. Cambridge, MA: MIT Press.

Geiger, Jeffrey \& Ming Xiang. 2019. Production of deaccenting under entailment, repetition, and bridging: Phonetic and perceptual comparisons. In Sasha Calhoun, Paola Escudero, Marija Tabain \& Paul Warren (eds.), Proceedings of 19th International Congress of Phonetic Sciences, Melbourne, Australia 2019, 512-516. Canberra, Australia: Australasian Speech Science and Technology Association Inc.

Lakoff, George. 1968. Pronouns and reference. In James D. McCawley (ed.), Syntax and Semantics 7: Notes from the Linguistic Underground, 275-335. New York: Academic Press. https://doi.org/10.1163/9789004368859_018.

Pierrehumbert, Janet B. \& Julia Hirschberg. 1990. The meaning of intonational contours in the interpretation of discourse. In Philip R. Cohen, Jerry Morgan \& Martha E. Pollack (eds.), Intentions in Communication, Cambridge, MA: MIT Press. https://doi.org/10.7551/mitpress/3839.003.0016.

Rochemont, Michael S. 1986. Focus in Generative Grammar. Amsterdam: John Benjamins. https://doi.org/10.1075/sigla.4.

Rooth, Mats. 1992. Ellipsis redundancy and reduction redundancy. In Steve Berman \& Arild Hestvik (eds.), Proceedings of the Stuttgart Ellipsis Workshop: Arbeitspapiere des Sonderforschungsbereichs 340, Stuttgart: Universitäten Stuttgart und Tübingen in Kooperation mit der IBM Deutschland.

Schwarzschild, Roger. 1999. Givenness, AvoidF and other constraints on the placement of accent. Natural Language Semantics 7(2). 141-177. https://doi.org/10.1023/A:1008370902407.

Selkirk, Elisabeth O. 1984. Phonology and Syntax: The Relation between Sound and Structure. Cambridge, MA: MIT Press.

Tancredi, Christopher. 1992. Deletion, deaccenting, and presupposition. Cambridge, MA: MIT dissertation.

Wagner, Michael. 2012. Focus and givenness: A unified approach. In Ivona Kučerová \& Ad Neeleman (eds.), Contrasts and Positions in Information Structure, 102-147. Cambridge: Cambridge University Press. https://doi.org/10.1017/CBO9780511740084.007. 